\title{
Resolution of Severe Left Ventricular Dysfunction and Development of Pulmonary Hypertension with Change of Thyroid Function from Hypothyroidism to Hyperthyroidism in a Patient with Thyrotoxicosis: A Case Report
}

\author{
Palangasinghe $\mathrm{DR}^{\mathrm{l} *}$, Kularathne $\mathbf{O A R}^{2}$ and \\ Herath HMM ${ }^{3}$ \\ ${ }^{1}$ Senior Registrar in Medicine, University Medical Unit, \\ Teaching Hospital Karapitiya, Sri Lanka \\ ${ }^{2}$ Registrar in Medicine, University Medical Unit, Teaching \\ Hospital Karapitiya, Sri Lanka \\ ${ }^{3}$ Senior Lecturer in Medicine, Consultant Physician, \\ Faculty of Medicine, University of Ruhuna, Sri Lanka \\ *Corresponding author: Palangasinghe DR, \\ University Medical Unit, Teaching Hospital Karapitiya, \\ Sri Lanka
}

Received: January 20, 2018; Accepted: February 21, 2018; Published: March 18, 2018

\begin{abstract}
Background: Both thyrotoxicosis and hypothyroidism could be associated with significant cardiovascular abnormalities. We report a patient with thyrotoxicosis who first developed reversible cardiomyopathy associated with hypothyroidism and then developed moderately severe pulmonary hypertension with hyperthyroidism. This transformation of cardiac status was coincided with the change of thyroid function from hypothyroidism to hyperthyroidism.
\end{abstract}

Case Presentation: This patient was a 56-year male with thyrotoxicosis who initially presented to us with anti-thyroid agents induced hypothyroidism associated with severe symptomatic congestive cardiac failure. Treatment with thyroxin leads to improvement of his clinical status and normalization of ejection fraction. However, two years later he presented to us with hyperthyroidism and features of pulmonary hypertensionand right heart failure. Repeat Echocardiogram revealed moderate to severe pulmonary hypertension and right ventricular dysfunctionwith normal left ventricular ejection fraction. With anti-thyroid medications, he had symptomatic improvement with reduction of pulmonary pressure.

Conclusion: Thyroid disorders could mainly present with profound cardiovascular dysfunction. Correction of underlying thyroid derangement itself results in resolution of associated cardiovascular dysfunction. Left ventricular systolic dysfunction secondary to severe hypothyroidism and pulmonary hypertension secondary to thyrotoxicosis are potentially reversible with the treatment of underlying thyroid disorder.

Keywords: Hypothyroidism; Left ventricular systolic dysfunction; Hyperthyroidism; Pulmonary hypertension

\section{Introduction}

Thyroid hormones have a key role on cardiovascular health. Both hyperthyroidism and hypothyroidism could lead to deranged cardiac functions. Hyperthyroidismleads to increase in resting heart rate, blood volume, stroke volume, myocardial contractility, and ejection fraction [1]. Hyperthyroidism could clinically manifest as sinus tachycardia, supraventricular premature contractions, atrial fibrillation, cardiac hypertrophy, systolic hypertension, thyrotoxic cardiomyopathy or congestive heart failure [2,3]. Hyperthyroidismcould also lead to isolated right heart failure, variable degrees of tricuspid regurgitation and pulmonary hypertension [2]. Hypothyroidism could also result incardiovascular dysfunction, which include sinus bradycardia, varying degrees of heart block and pericardial effusions [4]. Herein we report a patient who suffered severe cardiovascular dysfunction related to both hypo and hyperthyroidism at different times. Our patientwas diagnosed with thyrotoxicosis and while on antithyroid medications developed hypothyroidism with dilated cardiomyopathy which subsequently resolved with thyroxin replacement. He later became thyrotoxic again and developed severe pulmonary hypertension and right heart failure.Exact mechanism for the development of pulmonary hypertension in thyrotoxicosis is unclear. But various mechanisms had been postulated, such as enhanced catecholamine sensitivity causing pulmonary vasoconstriction, reduced pulmonary vascular compliance and an increased vascular resistance; increased metabolism of pulmonary vasodilating substances (prostacyclin, nitric oxide); decreased or impaired metabolism of pulmonary vascontrictors (serotonin, endothelin 1 and tromboxane) $[5,6]$. Achievement of euthyroid status would result in resolution of pulmonary hypertension in most of the time if the underlying cause is thyrotoxicosis as in our case.

\section{Case Presentation}

A 56 year old Sri Lankanmale patient with thyrotoxicosis and atrial fibrillation for 10 years while on carbimazole with defaulted follow up presented with lethargy, constipation and bilateral 
ankle swelling with progressive shortness of breath two years ago. At that time he was detected to have high thyroid hormone stimulating levels (TSH - $100 \mathrm{mU} / \mathrm{L}(0.3-4.0)$ ) and low Free T4 (T4-0.1 pmol/L (10-28)) and transthoracic echocardiogram showed evidencedilated cardiomyopathy with an ejection fraction of $15 \%$. In addition to carbimazole $10 \mathrm{mg}$ tds, he was on warfarin at that time. Carbimazole was discontinued and he was commenced on thyroxin $(75 \mu \mathrm{g})$ replacement therapy which he continued for 2 years with gradual improvement of his symptoms. He did not get a follow up echocardiogram or thyroid function tests during thosetwo years but he continued to take thyroxin and warfarin. While on thyroxin replacement therapy at a dose of $75 \mu \mathrm{g}$ daily, he presented with shortness of breath on exertion, palpitations, tremors and watery diarrhea for three weeks duration. He did not smoke cigarettes or consume alcohol. He had been a vegan for the last six years. He did not have any bleeding manifestations.

Clinical examination revealed that he was dyspneic, tachypneic, and pale with bilateral leg edema. He was irritable and had bilateral fine hand tremors. Cardiovascular system examination showed irregularly irregular pulse at a rate of $108 / \mathrm{min}$ with blood pressure of 100/70 $\mathrm{mmHg}$, elevated jugular venous pulse (JVP) with a CV wave, apical grade 3 pansystolic murmurs, loud second heart sound, parasternal heaving and a pulsatile liver.

Patient was clinically thyrotoxic which was confirmed biochemically by elevated free T4 level (96 pmol/L ( 10-28)) with low TSH levels $(<0.001 \mathrm{mU} / \mathrm{L}(0.4-4.0))$.Thyroxin replacement therapy was stopped on admission and he was commenced on oral frusemide $40 \mathrm{mg}$ mane with carbimazole $15 \mathrm{mg}$ tds. Once his heart failure was stable he was started on oral propanolol $40 \mathrm{mg}$ twice daily with carbimazole $15 \mathrm{mg}$ three times daily.Warfarin was continued because of persistent atrial fibrillation.

His investigations were as follows. Haemoglobin level was found to have $7.4 \mathrm{~g} / \mathrm{dL}$ with mean corpuscular volume (MCV)-83fL, mean corpuscular haemoglobin concentration (MCH)-26 Pg, and Mean corpuscular haemoglobin concentration $(\mathrm{MCHC})-32 \mathrm{~g} / \mathrm{dl}$, red cell distribution width (RDW) - 13.8\%. Platelet count was $122 \times 10^{3} /$ $\mathrm{mm} 3$ and white cell count was $6500 / \mathrm{mm}^{3}$ with neutrophils- $61 \%$ and lymphocytes-20\%. Blood picture revealed dimorphic red cellswith hypochromic microcytic cells as well as oval macrocyte with several hypersegmented neutrophils suggestive of mixed deficiency anaemia. Prothrombin time international normalized ratio (PT/INR) was 2.2 with normal activated partial thromboplastin time (APTT). As he had been a vegan with mixed deficiency anemia he was commenced on iron, folate, ascorbic acid and parenteral vitamin B12 replacement therapy. He was discharged on oral propanolol $40 \mathrm{mg}$ three times daily, carbimazole $15 \mathrm{mg}$ three times daily and warfarin $5 \mathrm{mg}$ daily. He was not commenced on any specific therapy for pulmonary hypertension.

Transthoracic echocardiogram revealed that normal left ventricle dimensions with ejection fraction (EF) of $60 \%$, mild to moderate mitral regurgitation (MR) and moderate to severe pulmonary hypertension with right ventricular dysfunction.High resolution computerized tomography of chest did not show any evidence of interstitial lung disease and venous duplex of lower limbs did not reveal any evidence of venous thrombosis.
Review of this patient in 3 months revealed that he was less symptomatic with improving dyspnea and ankle swelling and repeat echocardiogram showed improvement in pulmonary pressures and right ventricular functions. His thyroid functions revealed normal free T4 levels with low normal TSH levels (o.4) and haemoglobin level had improved up to $9.4 \mathrm{~g} / \mathrm{dL}$.

\section{Discussion}

Both hypothyroidism and hyperthyroidism results in cardiovascular dysfunction where patients with a thyroid disorder could primarily present with symptoms and signs related to that [1]. Good aspect of it is; most of thyroid disorder related cardiovascular dysfunctions are fully reversible with achievement of euthyroid state $[1,2]$. Our patient once had hypothyroidism related severe left ventricular failure which got fully reversed with thyroxin replacement therapy while subsequently developed severe right failure and pulmonary hypertension related to thyrotoxicosis which was getting resolved with improvement in thyroid functions. Our patient was inadvertently on carbimazole for 10 years because of defaulted follow up initially, went on to develop drug induced hypothyroidism which was detected two years ago. Hypothyroidism has resulted in severe left ventricular systolic dysfunction for which alternative causes were less likely since it got fully resolved with thyroid replacement therapy. Although cardiac output is reduced in hypothyroidism, heart failure is a rare presentation because there is a lower demand for peripheral oxygen delivery $[3,4]$. While on thyroid replacement therapy thyrotoxicosis recurred in our patient with associated severe pulmonary hypertension and right heart failure. Thyrotoxicosis was reported to be associated with pulmonary hypertension in many case reports, few observational studies and it is included under one of the category 5 causes (miscellaneous causes) of pulmonary hypertension in the WHO classification $[5,6]$. Thyroid function tests are important in evaluating a patient with pulmonary hypertension when there is no obvious underlying cause since the main clinical manifestation of thyrotoxicosis could be pulmonary hypertension and in most of the occasions it is reversible with achievement of euthyroid state [7]. In our patient alternative causes of pulmonary hypertension like interstitial lung disease and venous thromboembolism were excluded. More importantly improvement of thyrotoxicosis resulted in resolution of pulmonary pressures and right ventricular functions supporting a causal relationship between the two. In thyrotoxicosis following mechanisms possibly mediate the development of pulmonary hypertension. Increased catecholamine sensitivity resulting in pulmonary vasoconstriction, decrease metabolism of pulmonary vasoconstrictors and enhanced metabolism of pulmonary vasodilators. Even with above presumed mechanisms why pulmonary hypertension only manifests in certain individuals with thyrotoxicosis in not clear and probably a genetic susceptibility may explain it $[7,8]$. Other comorbid condition detected in our patient was severe mixed iron and vitamin B12 deficiency anemia which was treated with replacement therapy. He had been a vegan for the last 6 years with dietary inadequacy of both iron and vitamin B12 could have resulted in this deficiency state. Although there were no reported associations between nutritional anemias and pulmonary hypertension; chronic hemolytic anemias, hematological disorders like polycythemia vera and essential thrombocythemia were known to be associated with pulmonary hypertension [9]. 
Our patient had severe cardiovascular dysfunction related to both hypothyroidism and hyperthyroidism during the course of his illness at different times. When he was detected to have hypothyroidism there was evidence of severe left ventricular systolic dysfunction which got reversed with thyroid replacement therapy. When he presented with recurrence of thyrotoxicosis there was evidence of severe pulmonary hypertension with right heart failure which got corrected with antithyroid medication treatment and achievement of euthyroid state. Both the severe left ventricular dysfunction with an ejection fraction of $15 \%$ and severe pulmonary hypertension with right heart failure are conditions associated with high morbidity and mortality. However both the times the treatment of underlying thyroid dysfunction resulted in resolution of the condition. This case highlights the importance of searching and optimally treating an underlying thyroid disorder in a patient with potentially life threatening cardiovascular disease like severe left ventricular failure and pulmonary hypertension.

\section{Consent}

Written informed consent was obtained from the patient for the publication of this case report.

\section{References}

1. Vargas-Uricoechea $\mathrm{H}$, Bonelo-PerdomoA, Sierra-Torres $\mathrm{CH}$. Effects of thyroid hormones on the heart. Clin Investig Arterioscler. 2014; 26: 296-309.
2. Al-Ghamdi AS, Aljohani N. Graves' thyrotoxicosis-induced reversible cardiomyopathy: a case report. Clin Med Insights Case Rep. 2013; 6: 47-50.

3. Iryna Tsymbaliuk, Dmytro Unukovych, Nataliia Shvets, Andrii Dinets. Cardiovascular Complications Secondary to Graves' disease: A Prospective Study from Ukraine. PLOS. 2015.

4. Stănescu C, Branidou K, Ranetti EA. Heart failure and dilated cardiomyopathy associated with severe longstanding untreated hypothyroidism. Rom J Intern Med. 2007; 45: 77-83.

5. Ryan JJ, Thenappan T, Luo N, Ha T, Patel AR, Rich S, et al. Archer. The WHO classification of pulmonary hypertension: A case-based imaging compendium. Pulm Circ. 2012; 2: 107-121.

6. Vallabhajosula S, Radhi S, Cevik C, Alalawi R, Raj R, Nugent K. Hyperthyroidism and pulmonary hypertension: an important association. Am J Med Sci. 2011; 342: 507-512.

7. Silva DR, Gazzana MB, John AB, Siqueira DR, Maia AL, Barreto SS. Pulmonary arterial hypertension and thyroid disease. J Bras Pneumol. 2009. 179-185.

8. Marvisi M, Balzarini L, Mancini C, Mouzakiti P. Thyroid gland and pulmonary hypertension. What's the link? Panminerva Med. 2013; 55: 93-97.

9. Simonneau G, Robbins VM, Beghetti M, Channick RN, Delcroix M, Denton $\mathrm{CP}$, et al. Updated Clinical Classification of Pulmonary Hypertension; journal of the American College of Cardiology. 2009; 54: 43-54.
Austin J Clin Case Rep - Volume 5 Issue 1 - 2018

ISSN : 2381-912X | www.austinpublishing group.com

Palangasinghe et al. (C) All rights are reserved
Citation: Palangasinghe DR, Kularathne OAR and Herath HMM. Resolution of Severe Left Ventricular Dysfunction and Development of Pulmonary Hypertension with Change of Thyroid Function from Hypothyroidism to Hyperthyroidism in a Patient with Thyrotoxicosis: A Case Report. Austin J Clin Case Rep. 2018; 5(1): 1129. 